\title{
A comparison of two methods of quantifying masticatory pressures developed under dentures with variable occlusal widths
}

\author{
WILLIAM H. ROEDEMA \\ The University of Michigan, School of Dentistry, Ann Arbor, Michigan
}

\section{Summary}

Pressures developed during mastication with removable dental prostheses have been quantified by two methods. This pilot study (1) compares the peak pressure method of pressure quantification with the integrated curve method of quantification and (2) continues the exploration of the effect of the width of the occlusal table on pressures developed under dentures.

A set of specially designed experimental dentures was constructed for one individual. The mandibular experimental denture contained a series of pressure transducers by which masticatory pressures were recorded. These pressures were monitored between the denture base and the residual ridge tissues. Several interchangeable posterior occlusal segments, varying only in the width of the occlusal table, were fabricated for the opposing maxillary denture.

Masticatory pressure and total ridge pressure were quantified by the integrated curve method and the peak pressure method. Both methods indicated a direct relationship between masticatory pressures produced and the width of the occlusal table. A similar direct relationship between total ridge pressure and occlusal width was suggested statistically by the integrated curve method, and graphically by both methods.

\section{Introduction}

Masticatory pressures developed under removable dental prostheses may be influenced to some degree by the artificial replacement teeth they contain. The occlusal surfaces of artificial posterior replacement teeth may be characterized by material, shape or configuration, and area. The direct relationship between the area of the occlusal table and forces or pressures developed during mastication has been supported by the investigations of Brudevold (1951), Karies (19.56, 1958), Frechette (1955), Bearn $(1971,1973)$ and Roedema (1976).

Two basic approaches to quantification of masticatory forces or pressures have been used in these investigations. The first method considered peak or maximum forces or pressures per stroke, and their means (Brudevold, 1951; Karies, 1956, 1958; Frechette, 1955; Bearn, 1971, 1973). The sum of the peak values for each stroke in the masticatory

Correspondence: Dr William H. Roedema, The University of Michigan, School of Dentistry, Complete Denture Department, Ann Arbor, Michigan 48109, U.S.A.

0305-182X/79/0100-0067 \$02.00 (C) 1979 Blackwell Scientific Publications 
sequence was considered an indication of the total force or total pressure applied to the crest of the residual ridge (Brudevold, 1951; Frechette, 1955; Bearn, 1971, 1973). A second approach was reported of a pilot study which related the entire pressure curve recorded during mastication to the buccolingual dimension of the occlusal table (Roedema, 1976). In this study, average pressures were computed for each masticatory sequence by integrating the pressure curves recorded from individual pressure transducers and dividing these values by the duration of the masticatory sequence. The product of mean pressure and duration of the masticatory sequence was considered an indication of total pressure developed at the ridge crest.

This report of the same pilot project continues to evaluate the relationship between the width of the occlusal table and pressures developed under dentures. The specific objectives of this report are:

(1) To evaluate and compare masticatory pressures calculated by two methods of quantification, i.e. the integrated curve method and the peak pressure method.

(2) To evaluate and compare total ridge pressure calculated by two methods of quantification, i.e., the integrated curve method and the peak pressure method.

\section{Methods and materials}

A set of specially designed experimental dentures was constructed for one individual (Fig. 1). Four interchangeable posterior occlusal segments, varying only in the width of the occlusal table, were fabricated for the maxillary denture. The mandibular experimental denture contained a series of pressure transducers by which pressures developed between the denture base and the subject's residual ridge were recorded.

The recorded pressure pulses were quantified by the integrated curve method and by the peak pressure method. The effect of the width of the occlusal table was observed graphically and statistically.

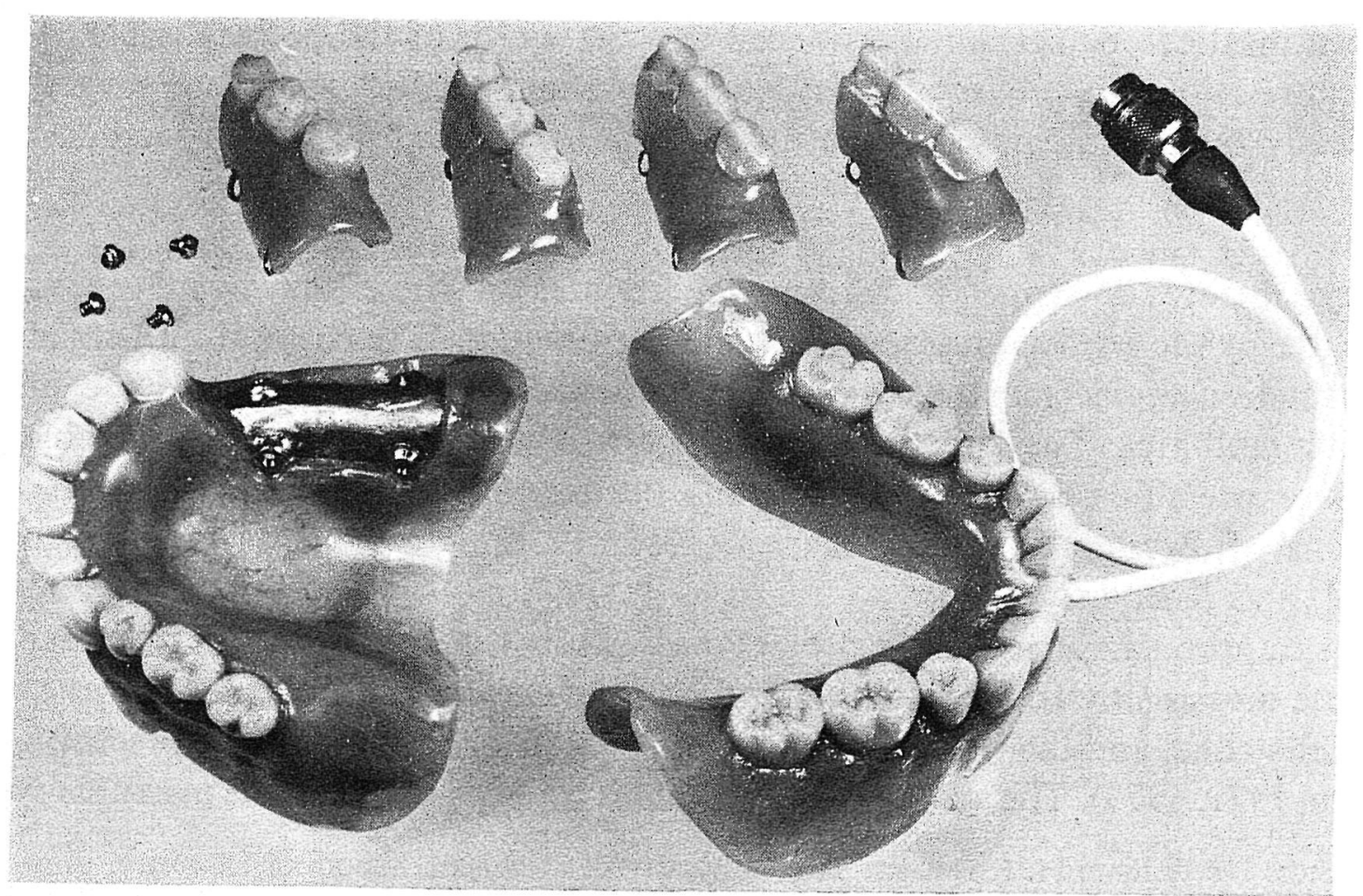

Fig. 1. The experimental gold base maxillary and mandibular dentures with the four experimental occlusal tables. 
The subject recorded 96 masticatory sequences, i.e. forty-eight sequences on each of two days of testing. Each day carrot segments $(1 \times 1 \times 2 \mathrm{~cm})$ were the test food in twenty-four of the sequences, and raisin masses (four raisins pressed together), in the other twenty-four. Four occlusal segments varying only in the width of the occlusal table were tested on each day (Fig. 2). On the first day, the testing began with the narrowest occlusal segment, occlusal width (O.W.) IV. The other occlusal widths were introduced and reintroduced in the following order: O.W. III, O.W. II, and O.W. I, followed by O.W. I, O.W. II, O.W. III, and O.W. IV. During the next test session, the sequence of introduction of the experimental occlusal tables was from the widest to
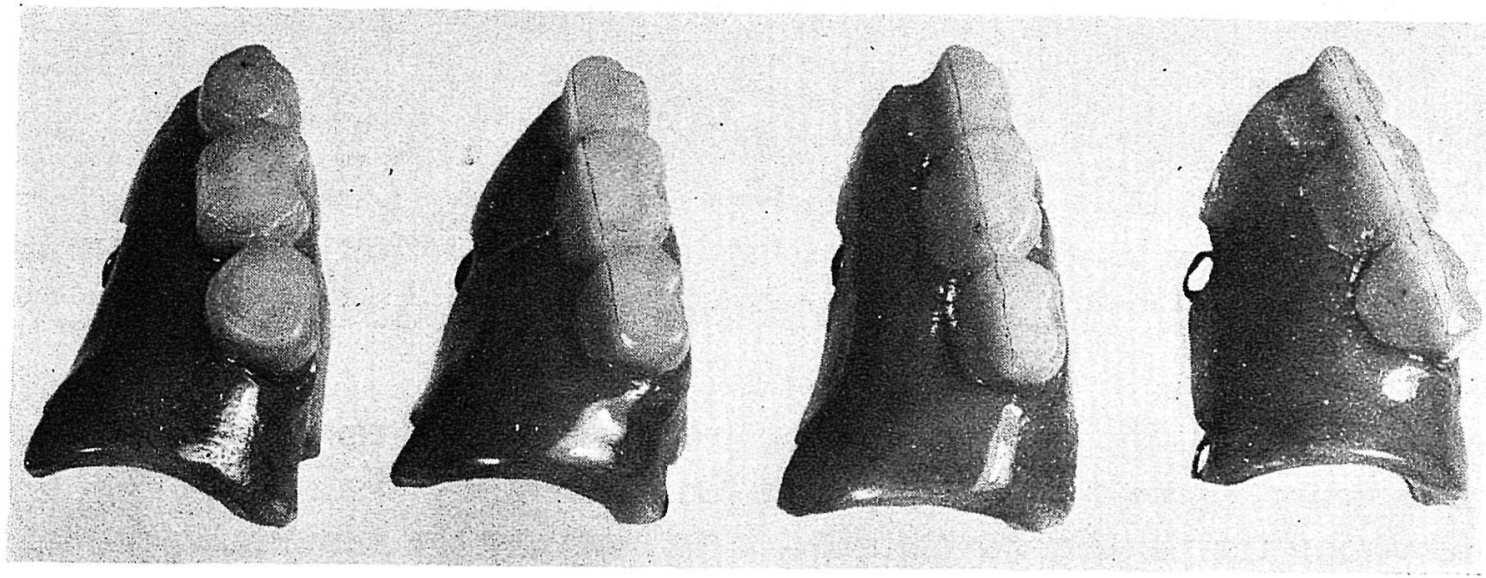

Fig. 2. The four experimental occlusal segments, from left to right, Occlusal width (O.W.) I, O.W. II, O.W. III, and O.W. IV.

the narrowest (O.W. I, O.W. II, O.W. III, and O.W. IV) and then from the narrowest to the widest occlusal form (O.W. IV, O.W. III, O.W. II, and O.W. I). Three complete masticatory sequences were recorded using carrots, followed by three complete sequences using raisins, for each occlusal segment introduced. Thus, on each day for each test food, there were recorded six masticatory sequences for each occlusal table width.

The recording sites selected for analysis were three of the four along the crest of the subject's residual ridge. The three selected were located under the bicuspid, first molar, and second molar, and were respectively labelled R1, R2, and R3 (Fig. 3). Since the fourth recording site on the crest of the ridge had not been located under a tooth, but had been placed distal to the second molar, it was not considered in this analysis. The pressure sensing devices were diaphragm pressure transducers, identical to those reported by Kelsey, Reid \& Coplowitz (1976).

\section{Quantification of masticatory pressures}

The pressure curve of each masticatory sequence was quantified by the integrated curve method and by the peak pressure method. The values obtained from each method were subjected to identical statistical analyses. The results of these analyses were compared.

Integrated curve method of quantification. An average pressure was determined at each of the three recording sites for each masticatory sequence. Each of the pressure pulses, recorded* on magnetic tape during the pilot study, was converted to numerical

* Hewlett Packard model No. 3955 instrumentation tape recorded, Hewlett-Packard Company, Palo Alto, California, U.S.A. 


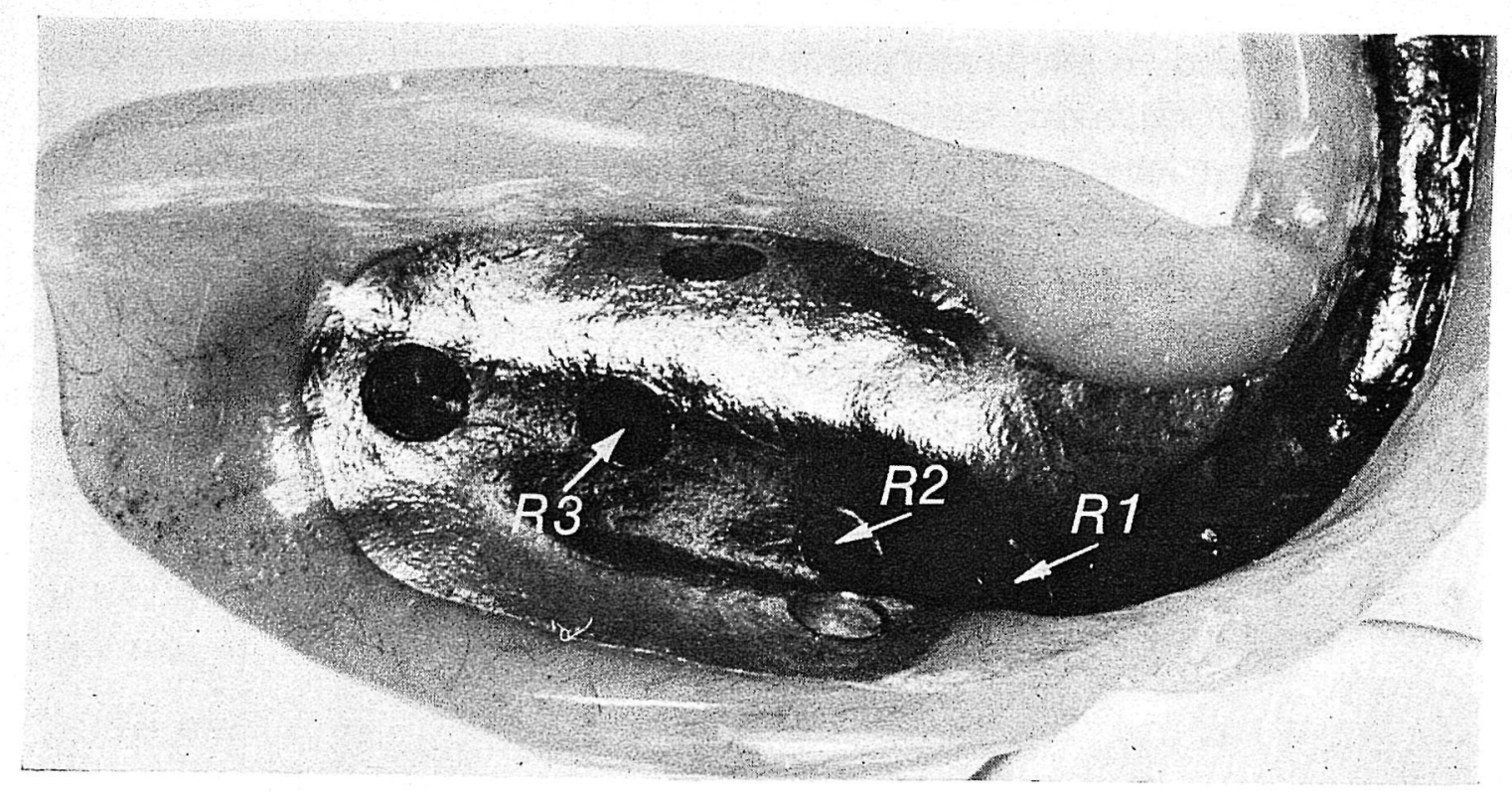

Fig. 3. The tissue surface of the mandibular experimental denture on the subject's preferred chewing side, showing the location of the three pressure transducers (R1, R2 and R3) selected for analysis.

display* and integrated. $\uparrow$ The integrated values were divided by the duration of the masticatory sequence. These computations resulted in average pressure.

An expression of the pressure developed along a length of the mandibular residual ridge was computed by averaging the individual average pressures from $\mathrm{R} 1, \mathrm{R} 2$, and $\mathrm{R} 3$ for each masticatory sequence. This combination of pressures from three recording sites is known as mean pressure.

Thus, by convention, the word average is used to describe pressure at individual recording sites; the word mean is used to describe the combined pressure at multiple recording sites.

Peak pressure method of quantification. The Thompson P.F. 40 Digitizerł was used to record from the original oscillograph readouts§ the maximum or peak pressures developed during each masticatory stroke (Fig. 4). For each masticatory sequence the values of the individual strokes were averaged to produce an average peak pressure for each recording site. The mean peak pressure of each sequence was obtained by combining the average peak pressures from R1, R2, and R3.

Statistical analysis. Statistical analyses were made to determine the effects of the four occlusal widths on the recorded pressures. The statistical tests consisted of univariate analyses of variance of the average and average peak pressure values by recording site. Similar analyses were made of the mean and mean peak pressure values. Separate analyses of pressures were made for each day and for each test food.

Correlation coefficients were computed, by recording site, to directly compare average pressures and the corresponding average peak pressures. Similar computations

* Digital Equipment model AF 01-A, A-D Converter, Digital Equipment Corporation, Maynard, Massachusetts, U.S.A.

$\dagger$ Digital Equipment, model PDP 8/X digital computer, Digital Equipment Corporation, Maynard, Massachusetts, U.S.A.

$\$$ Instronics, Ltd., Stittsville, Ontario, Canada.

$\S$ Brush model No. 480 oscillograph with model No. 13-4312 direct current bridge amplifiers, Gould Incorporated, Brush Instrument Division, Cleveland, Ohio, U.S.A. 


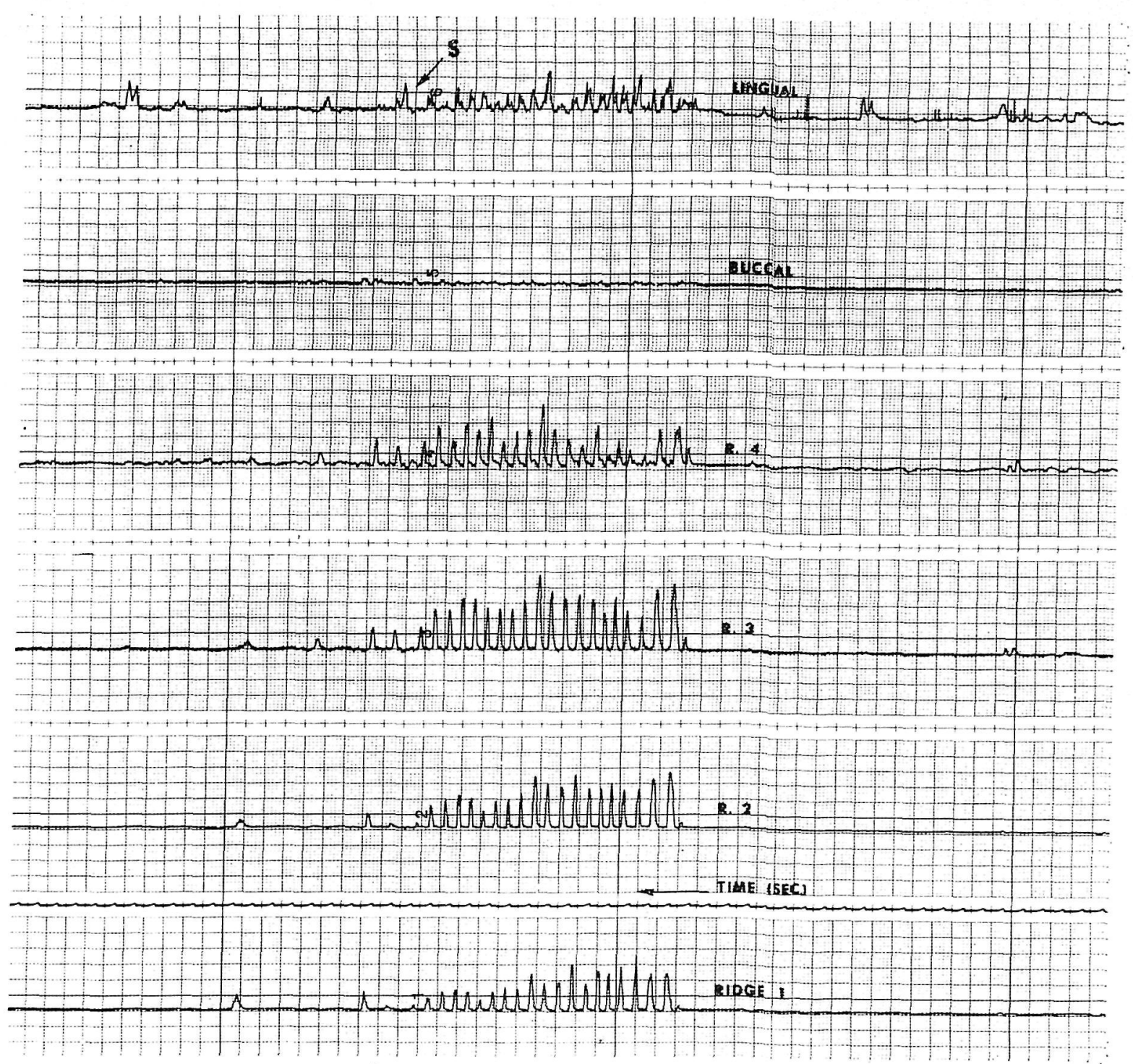

Fig. 4. Typical oscillograph readout of the pressures produced while chewing a mass of raisins with O.W. I. S indicates the time that swallowing begins.

were made to compare mean pressures with mean peak pressures for each occlusal width.

\section{Quantification of total masticatory pressures}

Integrated curve method of quantification. The average pressures and the mean pressures for each masticatory sequence were multiplied by the duration of that sequence to give an indication of the total pressure applied to the ridge during mastication of each sample food. The products of these two factors are reported as the average total and the mean total pressures.

Peak pressure method of quantification. Average total peak and mean total peak pressures were obtained by multiplying the average peak and the mean peak pressures by the number of chewing strokes in each masticatory sequence.

Statistical analysis. Univariate analyses of variance were made using the average and mean expressions of total pressure to determine the effect of the four occlusal widths. 
Number of strokes in masticatory sequence and duration of masticatory sequence Analyses of variance were also performed to determine the effect of occlusal width on the number of masticatory strokes required before swallowing and on the duration of the masticatory sequences.

\section{Results}

\section{Masticatory pressures}

Mean pressures. Mean masticatory pressures decreased with a reduction in the width of the occlusal table (Figs 5 and 6). This reduction in pressures occurred with both test foods on both days. The analyses of variance confirmed these observations for both carrots and raisins. Trends at the individual sites were generally similar. In only one instance (recording site R2, Day II, Raisins) was a confidence level obtained which was less than $95 \%$.

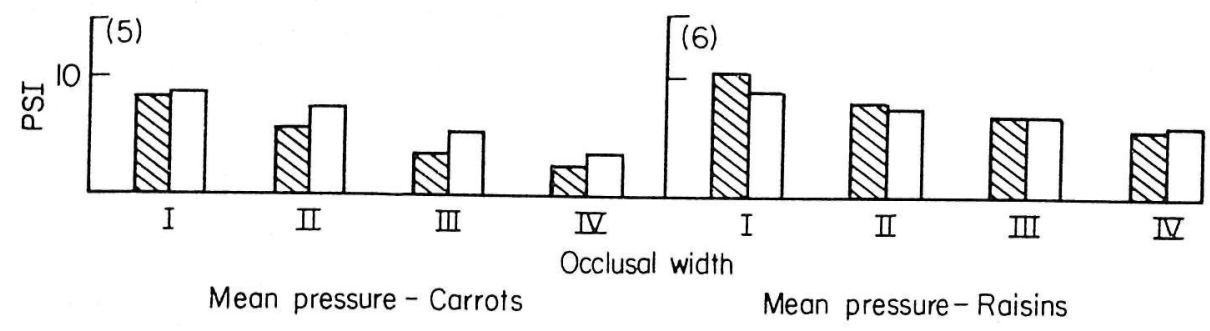

Fig. 5. The mean pressures developed at the crest of the mandibular residual ridge with the four experimental occlusal table widths using carrots. Hatched column, day $1(P<0 \cdot 0001)$; open columns, day $2(P<0 \cdot 0001)$.

Fig. 6. The mean pressures developed at the crest of the mandibular residual ridge with the four experimental occlusal table widths using raisins. Hatched columns, day $1(P=0 \cdot 0002)$; open columns, day $2(P=0 \cdot 0011)$.
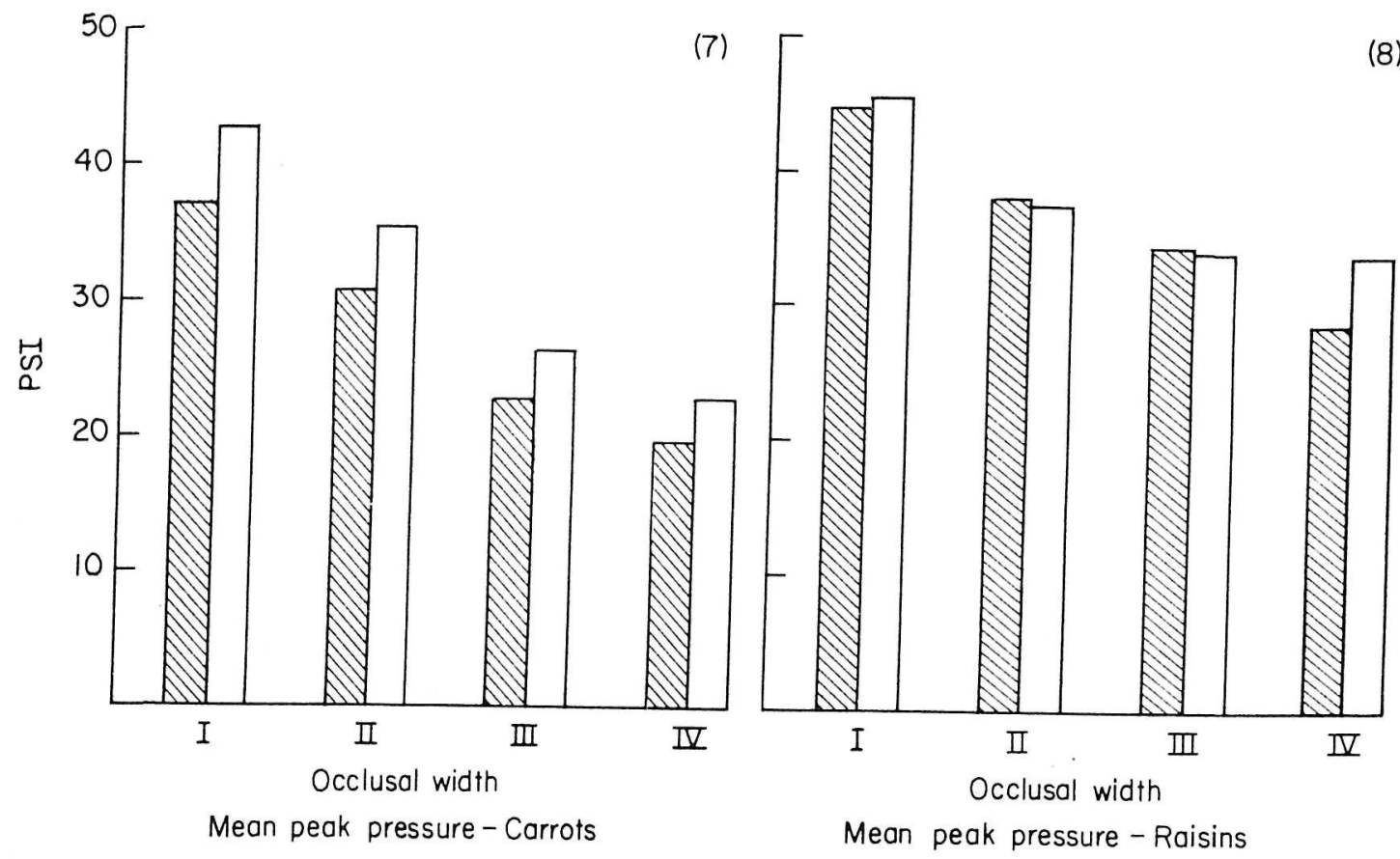

Fig. 7. The mean peak pressures developed at the crest of the mandibular residual ridge with the four experimental occlusal table widths using carrots. Hatched columns, day $1(P<0 \cdot 0001)$; open columns, day $2(P<0 \cdot 0001)$.

Fig. 8. The mean peak pressures developed at the crest of the mandibular residual ridge with the four experimental occlusal table widths using raisins. Hatched columns, day $1(P<0 \cdot 0001)$; open columns, day $2(P<0 \cdot 0001)$. 
Mean peak pressures. The graphic representations and statistical analyses of the relationship between the mean peak pressures and the four occlusal widths also indicated a direct relationship between pressure and buccolingual dimension of the occlusal tables (Figs 7 and 8). The average peak pressures showed a similar direct relationship. At each recording site the results were statistically significant, confidence levels being greater than $99 \%$.

Correlation between mean pressures and mean peak pressures. The correlation coefficients computed to compare the mean and mean peak pressures ranged between 0.8831 and 0.9792 (Table 1). At the individual sites, the correlation coefficients comparing average and average peak pressures were always positive, yet, never so high as those comparing the mean pressures.

Table 1. Correlation coefficients for mean pressure and mean peak pressure

\begin{tabular}{lll}
\hline Day 1 & Carrots & $0 \cdot 9792$ \\
& Raisins & $0 \cdot 9740$ \\
Day 2 & Carrots & $0 \cdot 9251$ \\
& Raisins & $0 \cdot 8831$ \\
\hline
\end{tabular}

\section{Duration of the masticatory sequence}

The effecis of the four occlusal widths on the duration of the masticatory sequence can be seen in Figs 9 and 10. Duration of the masticatory sequences generally varied inversely with the width of the occlusal table. The wider occlusal widths required shorter durations than the narrower widths. This inverse relationship was statistically significant with carrots as the test food. With raisins, however, the results were inconsistent.

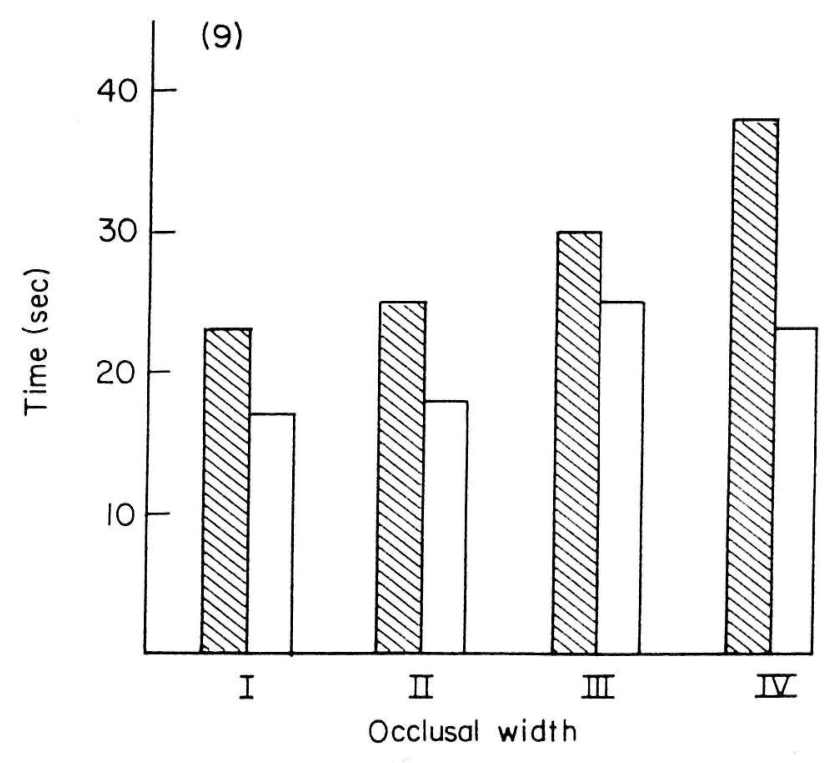

Duration of masticatory sequence-Carrots

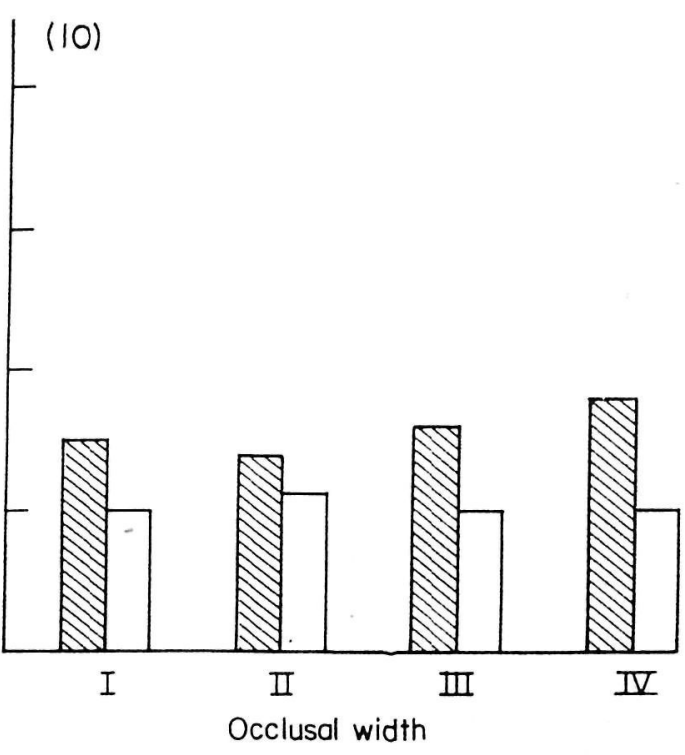

Duration of masticatory sequence - Raisins

Fig. 9. The mean durations of the masticatory sequences with the four experimental occlusal table widths using carrots. Hatched columns, day $1(P<0 \cdot 0001)$; open columns, day $2(P=0 \cdot 002)$.

Fig. 10. The mean durations of the masticatory sequences with the four experimental occlusal table widths using raisins. Hatched columns, day $1(P=0 \cdot 0118)$; open columns, day $2(P=0 \cdot 1843)$. 
Number of strokes in the masticatory sequences

Findings regarding the number of strokes required before swallowing are presented in Figs 11 and 12. Results of the statistical analyses indicated a significant inverse relationship between the number of masticatory strokes required before swallowing and the width of the occlusal table.
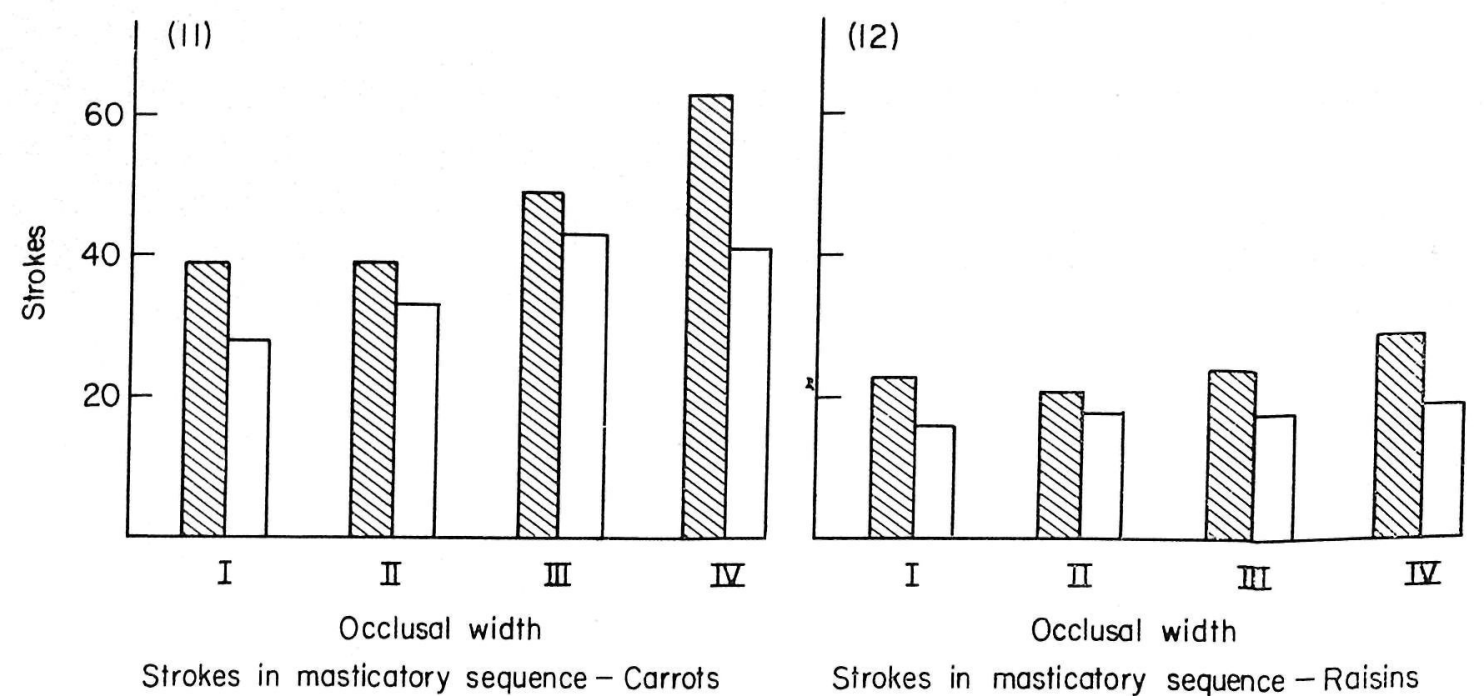

Fig. 11. The mean number of masticatory strokes in the masticatory sequences for the four experimental occlusal table widths using carrots. Hatched columns, day $1(P<0 \cdot 0001)$; open columns, day 2 $(P=0 \cdot 0001)$.

Fig. 12. The mean number of masticatory strokes in the masticatory sequences for the four experimental occlusal table widths using raisins. Hatched columns, day $1(P=0 \cdot 0141)$; open columns, day 2 $(P=0 \cdot 0193)$.

\section{Total masticatory pressures}

Mean total pressure. The mean total pressure at the crest of the residual ridge varied directly with the buccolingual width of the experimental occlusal tables for both test

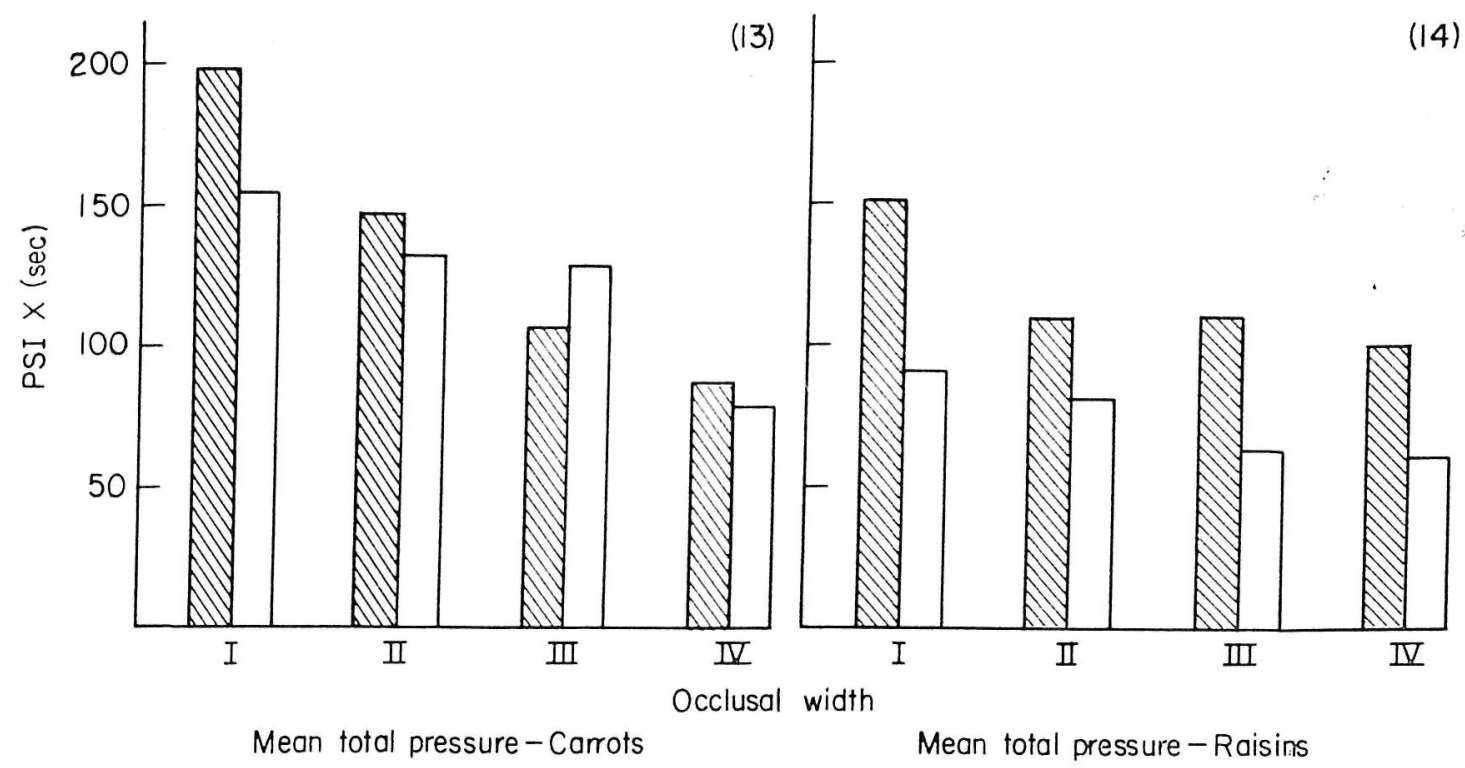

Fig. 13. The mean total pressures at the ridge crest during the corresponding masticatory sequences for the four experimental occlusal table widths using carrots. Hatched columns, day $1(P<0 \cdot 0001)$; open columns, day $2(P=0 \cdot 0219)$.

Fig. 14. The mean total pressures at the ridge crest during the corresponding masticatory sequences for the four experimental occlusal table widths using raisins. Hatched columns, day $1(P=0 \cdot 0432)$; open columns, day $2(P=0 \cdot 0075)$. 
foods (Figs 13 and 14). This observation was confirmed by statistical analysis. Less consistent findings were evident at the individual recording sites where confidence levels greater than $95 \%$ were found seven out of twelve times.

Mean total peak pressure. The relationship between the buccolingual width of the occlusal table and the mean total peak pressure was inconsistent (Figs 15 and 16). The statistical analyses did not reject the null hypothesis for either carrots or raisins on Day 1 or for carrots on Day 2. The average total peak pressures at the individual

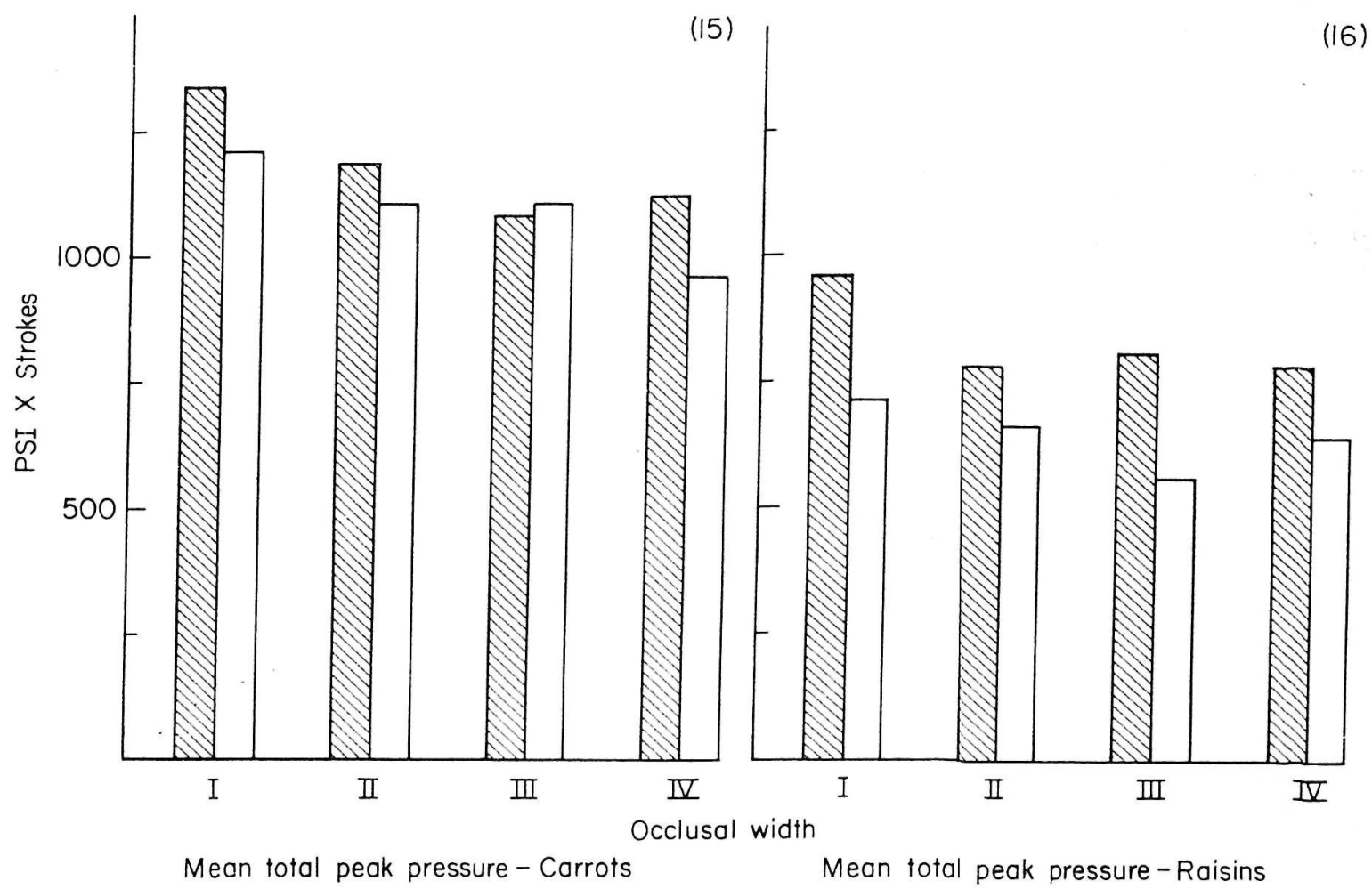

Fig. 15. The mean total peak pressures at the ridge crest during the corresponding masticatory sequences for the four experimental occlusal table widths using carrots. Hatched columns, day $1(P=0 \cdot 1189)$; open columns, day $2(P=0 \cdot 1671)$.

Fig. 16. The mean total peak pressures at the ridge crest during the corresponding masticatory sequences for the four experimental occlusal table widths using raisins. Hatched columns, day 1 $(P=0 \cdot 2652)$; open columns, day $2(P=0 \cdot 0174)$.

transducers were also related inconsistently to the width of the occlusal table. Confidence levels greater than $95 \%$ were found only four out of twelve times at the individual sites.

\section{Discussion}

Previous investigators have measured forces and pressures both within the denture (Brudevold, 1951; Bearn, 1971, 1973; Yurkstas \& Curby, 1953; Howell \& Brudevold, $1950)$ and at the interface between the supporting tissues and the dental prosthesis (Karies, 1956, 1958; Frechette, 1955; Roedema, 1976; Stromberg, 1955; Cutright et al., 1976). With one exception (Roedema, 1976) they recorded the peak force or pressure for each masticatory stroke. These peaks were averaged or summed to provide, respectively, average peak pressures or total peak pressures (Fig. 17). The analysis of peak pressures does not, however, consider the durations of the individual masticatory strokes (Fig. 18). A large peak pressure of long duration may be much more significant to the health of the residual ridge than the identical peak pressure of shorter duration. 
Peak pressure method

of quantification
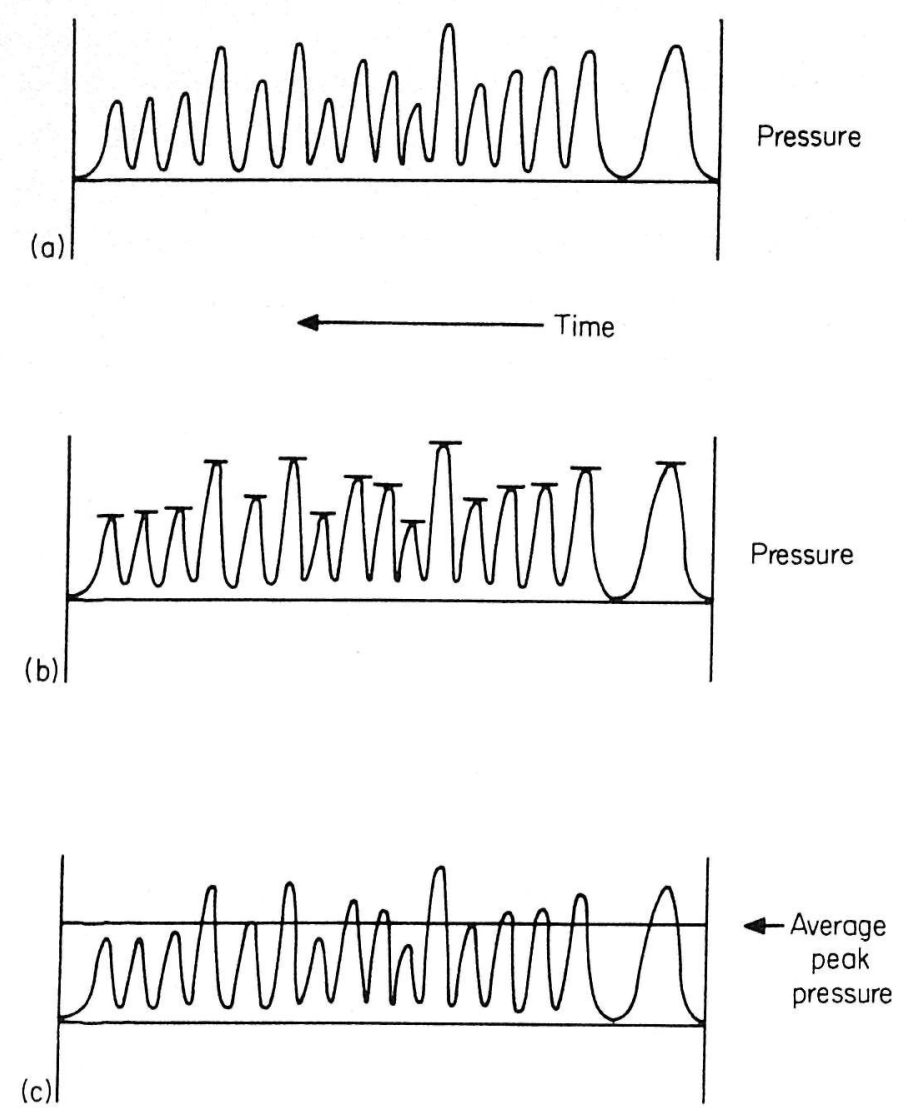

Fig. 17. The peak pressure method of quantitation: the average peak pressure for the masticatory sequence (a) is determined from the maximum pressures (b) developed during the individual masticatory strokes by averaging (c).

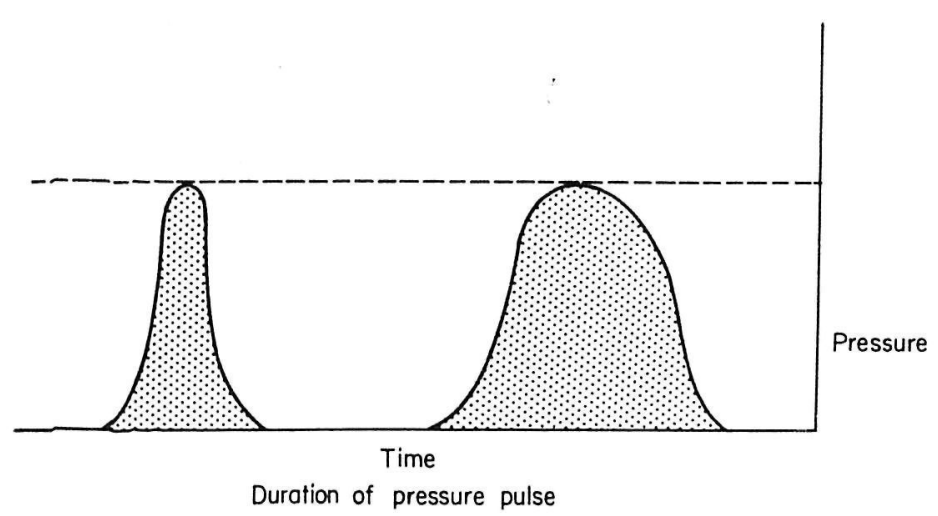

Fig. 18. Though masticatory strokes may be characterized by identical peak values, dissimilar durations may be significant to the health of the residual ridge tissues.

Roedema (1976) computed the average pressure by integrating the instantaneous pressure curve for each masticatory sequence and dividing by the duration of that sequence (Fig. 19). The magnitude of the pressure and its duration are both considered by the integrated curve method of pressure quantification. Integration basically determines the area under the curve, the product of the ordinate value (pressure) and the abscissa value (time). Dividing the integral of the pressure curve by the duration of the curve results in an average pressure value. An indication of the total pressure was 
Integrated curve method of quantification

(a)

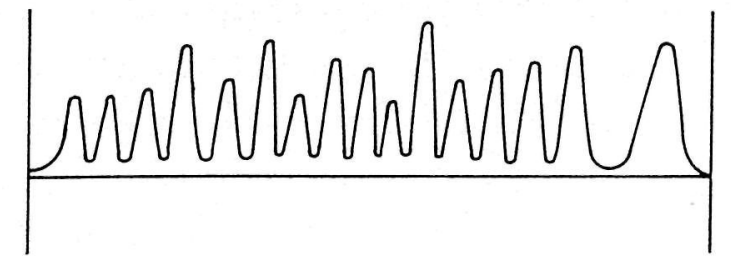

Pressure

(b)

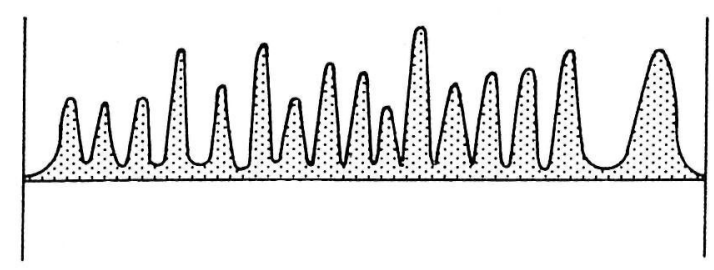

Pressure

(c)

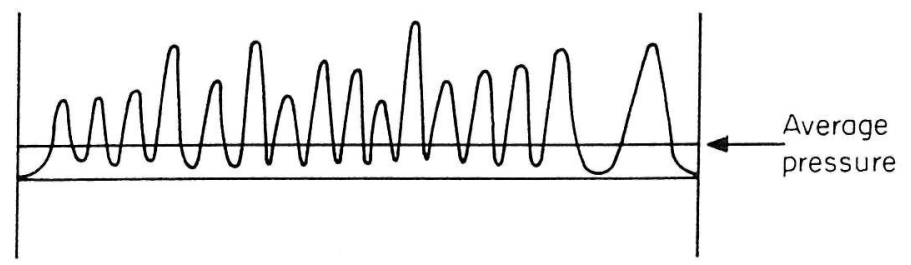

Fig. 19. The integrated curve method of quantitation: for the masticatory sequence (a), masticatory pressures are computed by dividing the area (b) (pressure $\times$ duration) under the entire pressure curve by the duration of the masticatory sequence resulting in average pressure (c).

obtained by multiplying the average or mean pressures for each masticatory sequence by the duration of that sequence.

\section{Statistical analysis}

The experiment's null hypotheses were established. Univariate analyses of variance were used to test the null hypotheses relating pressure and total pressures to the width of the occlusal table. Correlation coefficients were computed to compare mean pressures with mean peak pressures.

\section{Masticatory pressures}

To obtain an indication of the mean masticatory pressure developed along the crest of the residual ridge during a masticatory sequence, the average pressures recorded from the three recording sites were averaged. Correspondingly, mean peak pressures were obtained as the mean of the average peak pressures from the individual sites. The results were subjected to univariate analyses of variance for each of the two test foods on each test day. Highly significant statistical differences were found for both the mean pressures and the mean peak pressures produced with the various occlusal widths using both carrots and raisins (Figs 5-8). Furthermore, high positive correlation coefficients ranging from 0.8831 to 0.9790 were found relating mean pressure to mean peak pressure (Table 1). 
These results would seem to indicate that either mean pressures or mean peak pressures could be used with high levels of confidence to determine differences in pressures caused by alterations in occlusal widths, in the range of widths used.

With but one exception, confidence levels greater than $95 \%$ were computed for all average pressures and all average peak pressures at the individual recording sites. The exception occurred at the recording site $\mathrm{R} 2$, where the confidence level describing average pressures on Day 2 using raisins was less than $95 \%$. No explanation is apparent for this inconsistency. Even in this instance, however, the trend of greater average pressures with the wider occlusal widths was graphically evident. Though not so consistently high as those comparing mean and mean peak pressures, positive correlation coefficients were also found to relate the average and average peak pressures at the individual recording sites.

From the results of these statistical analyses, one may reasonably conclude that the analysis of the average of several recording sites along the ridge crest might be safer than the analysis of a single site. However, if a sufficiently large number of masticatory sequences were recorded and analysed, the number of recording sites might safely be reduced. As an overall picture of pressures over the entire ridge, however, several recording sites located along the entire crest would no doubt provide a more accurate representation of real occurrences. This may be especially noteworthy if one considers that food of various consistencies may be masticated in different sections of the posterior occlusal segments.

\section{Number of strokes in masticatory sequence and duration of masticatory sequence} The duration of the masticatory sequence generally increased with narrower occlusal tables (Figs 9 and 10). The number of masticatory strokes consistently increased with narrower occlusal tables (Figs 11 and 12). The patient was instructed to chew primarily on his preferred chewing side and to swallow when he normally would do so. No further instructions were given. Mastication with narrower teeth took longer and required more strokes than mastication with the wider teeth.

\section{Total masticatory pressures}

Mean total pressure. The mean total pressure has been defined as the product of the mean pressure and the corresponding duration of the masticatory sequence (Roedema, 1976). The product of these two factors may be considered an indication of the total pressure developed between the denture base and the residual ridge during a masticatory sequence. With a confidence level of greater than $95 \%$, the mean total pressure was found to vary directly with the buccolingual width of the occlusal table. Though the duration of the masticatory sequences increased with narrower occlusal tables, the total pressure applied to the residual ridge crest decreased.

When the average total pressures for the individual recording sites were subjected to similar statistical analyses, similar trends were evident. However, the high degree of statistical significance seen with the combination of the three recording sites was not consistently present for the individual sites. These findings may indicate that with occlusal tables of varying size, the anterio-posterior location of the masticatory effort may also vary.

Mean total peak pressure. The analyses of mean total peak pressures were inconclusive. Though the histograms appear to present a trend toward a direct relationship between 
mean total peak pressures and the width of the occlusal table, the experiment's null hypothesis was not consistently rejected by statistical means (Figs 14 and 15). Only on day 2 , with raisins as the test food, was the null hypothesis rejected at a confidence level of greater than $95 \%$. When the average total peak pressures from the individual pressure transducers were analysed, statistically significant differences were found for the various buccolingual widths in only four of twelve analyses.

An explanation of these results might be found in the relationship between the buccolingual dimension of the occlusal table and the components of mean total peak pressure. The number of masticatory strokes required before swallowing is related inversely to the width of the occlusal table. The mean peak pressure is directly related. One has the effect of offsetting the other in computation of mean total peak pressure, the product of these two factors.

Correlation between mean total pressures and mean total peak pressures. Since there were no consistent significant differences when mean total peak pressures were analysed, no correlation coefficients were computed to compare these values with the statistically significant mean total pressures.

The analyses of the mean total pressures and the mean total peak pressures at the surface indicates incompatible results. It would seem from the integration method of pressure computation that total pressures applied to the residual ridge crest might be reduced by reducing the width of the occlusal table. On the other hand, the findings from the peak pressure method do not support this conclusion.

A measure of caution must, however, be used in comparing these expressions of total masticatory pressure. The factors composing mean total pressure and mean total peak pressure must be carefully considered. Since mean total pressure has the units pressure $\times$ time, and mean total peak pressure has the units pressure $\times$ strokes, the two parameters are not identical and should not be directly compared.

The full significance of these findings will probably not be resolved until scientific studies can provide a definite casual relationship between magnitude or duration of pressure and destruction or stimulation of the residual alveolar ridges. Nevertheless, especially where the supporting residual ridge is diminutive or the tissues are friable, it would seem prudent to select narrower artificial replacement teeth and minimize the tendency to overstress the supporting tissues.

\section{Summary and conclusions}

Masticatory pressures can be quantified by the integrated curve method and by the peak pressure method. The masticatory pressures produced at the interface between the denture and its supporting residual ridge tissues were computed by these two methods for one subject. The results allow the following summarizing statements:

(1) Both the mean pressure and the mean peak pressure at the crest of the residual ridge varied directly with the width of the occlusal table.

(2) A high positive correlation existed between mean pressure and mean peak pressure within the range of the buccolingual widths of the occlusal tables tested.

(3) The mean total pressure at the crest of the residual ridge varied directly with the width of the occlusal table; the corresponding mean total peak pressure varied inconsistently with the width of the occlusal table.

(4) The number of masticatory strokes required before swallowing varied inversely with the width of the occlusal table. 
(5) The duration of the masticatory sequence generally varied inversely with the width of the occlusal table.

(6) A single recording site located under the supplied posterior teeth may not consistently provide the same quality of information concerning masticatory pressures that a series of recording sites may provide.

\section{Acknowledgments}

The author wishes to acknowledge the continued able support and constant encouragement of Dr Charles C. Kelsey and of Joel A. Coplowitz, especially during the initial phases of this project.

This investigation was supported by the Dental Research Institute at the University of Michigan, School of Dentistry, United States Public Health Service grant No. DE 02731, and by U.S.P.H.S. research grant no. 5501 RR 05321 from the General Research Service Branch, Division of Research Facilities and Resources.

\section{References}

BEARN, E. M. (1971) A preliminary report on a hydraulic measuring device for the study of forces transmitted by dentures. Dental Practitioner, 22, 17.

BEARN, E. M. (1973) Effect of different occlusal profiles on the masticatory forces transmitted by complete dentures: an evaluation. British Dental Journal, 134, 7.

BRudevold, F. (1951) A basic study of the chewing forces of a denture wearer. Journal of the American Dental Association, 43, 45.

Cutright, D. E., BrudviK, J. S., Gay, W. D. \& Selting, W. J. (1976) Tissue pressure under complete maxillary dentures. Journal of Prosthetic Dentistry, 35, 160.

FRECHETTE, A. R. (1955) Masticatory forces associated with the use of various types of posterior teeth. Journal of Prosthetic Dentistry, 5, 252.

Howell, A. H. \& BRudevold, F. (1950) Vertical forces used during chewing of food. Journal of Dental Research, 29, 133.

Karies, A. K. (1956) Partial denture design and its relation to force distribution and masticatory performance. Journal of Prosthetic Dentistry, 6, 672.

KarIES, A. K. (1958) A study of partial denture design and masticatory pressures in a mandibular bilateral distal extension case. Journal of Prosthetic Dentistry, 8, 340.

Kelsey, C. C., ReID, F. D. \& Coplowitz, J. A. (1976) A method for measuring pressures against supporting tissues under functioning complete dentures. Journal of Prosthetic Dentistry, 35, 376.

Roedema, W. H. (1976) Relationship between the width of the occlusal table and pressures under dentures during function. Journal of Prosthetic Dentistry, 36, 24.

StromberG, W. R. (1955) A method of measuring forces of denture base materials against supporting tissues. Journal of Prosthetic Dentistry, 5, 268.

Yurkstas, A. \& Curby, W. A. (1953) Force analysis of prosthetic appliances during function. Journal of Prosthetic Dentistry, 3, 82.

Manuscript accepted 7 September 1977 
This document is a scanned copy of a printed document. No warranty is given about the accuracy of the copy. Users should refer to the original published version of the material. 